\title{
Aortoesophageal fistula as a complication of thoracic aorta stent graft implantation: two cases and literature review
}

\author{
Olga Basiak', Laretta Grabowska-Derlatka², Tomasz Jakimowicz ${ }^{3}$, Olgierd Rowiński² \\ 'Students Scientific Association at the $2^{\text {nd }}$ Department of Clinical Radiology Medical University of Warsaw, Poland \\ ${ }^{2} 2^{\text {nd }}$ Department of Clinical Radiology, Medical University of Warsaw, Warsaw, Poland \\ ${ }^{3}$ Department of General, Vascular and Transplant Surgery, Medical University of Warsaw, Poland
}

\begin{abstract}
Thoracic endovascular aortic repair (TEVAR) is a method of choice in the treatment of thoracic aorta aneurysms and dissections. In case of a thoracoabdominal aneurysm, endovascular treatment is also being chosen more often, especially in patients with multimorbidity. Despite better results and less invasiveness in comparison to classic open surgery, endovascular treatment is also associated with complications. One of the rarer and usually fatal complications are aortoesophageal fistula (AEF). We present two cases, in which TEVAR complication was AEF. Case I was an 87-year-old woman with a history of TEVAR 5 years earlier, who presented increased inflammation parameters, massive gastrointestinal bleeding, and progressive anemia. Case 2 was a 66-year-old woman with a history of TEVAR 6 months earlier, who on admission presented medium increased inflammatory markers and anemia. None of the patients was qualified for surgical treatment. Both patient I and patient 2 died during hospitalization. Diagnostic imaging plays a key role in the diagnosis of AEF. CT angiography performed in patients with AEF can show the presence of gas in the sac of aneurysm as a result of infection, a defect in the aortic wall, or thickened esophagus with fluid level. CT angiography of the aorta combined with esophagogastroduodenoscopy (EGD) and contrast-enhanced X-ray examination of the gastrointestinal tract, enables to confirm or exclude the diagnosis of AEF. Atypical clinical feature and increased parameters of inflammation in patients with the history of TEVAR should always suggest the presence of AEF.
\end{abstract}

Key words: computer tomography, aortoesophageal fistula, aortobronchial fistula esophagogastroduodenoscopy, thoracic endovascular aortic repair

Acta Angiol 2020; 26, 2: 76-80

\section{Introduction}

Thoracic endovascular aortic repair (TEVAR) is a method of choice in the treatment of thoracic aorta aneurysms and dissections. In case of thoracoabdominal aneurysm, endovascular treatment is also being chosen more often, especially in patients with multimorbidity. Despite better results and less invasiveness in comparison to classical open surgery, endovascular treatment is also associated with complications. The most common complications are endoleaks to the aneurysmal sac. Rare complications include migration of stent graft, spinal cord ischemia and aortoesophageal fistula (AEF) [I]. We present two cases after thoracic stent graft implantation. We also review the literature.

\section{Case report I}

87-year-old woman, who had undergone stent graft implantation due to thoracic aortic aneurysm, was admitted to the hospital with weakness and dehydration. On admission the patient was in medium condition, and hemodynamically stable. Laboratory test results 


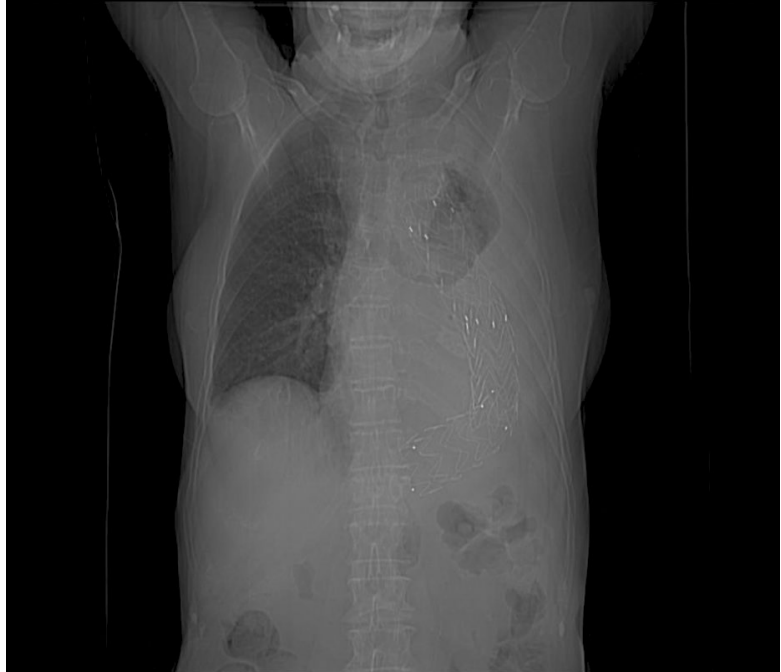

Figure I. Case report I. Chest radiograph showing pleural effusion and atelectasis of left lung

showed anemia $\left(\mathrm{Hb} 9,4 \mathrm{~g} / \mathrm{dl}\right.$, RBC 3,27 $\left.\times 10^{6} \mu \mathrm{l}\right)$, increased inflammatory markers (WBC 17,05 $\times 10^{6} \mu$, CRP $143,8 \mathrm{mg} / \mathrm{dl}$ ) and extremely elevated D-dimer $(5477 \mathrm{ng} / \mathrm{dl})$. Chest radiography showed pleural effusion and atelectasis of the left lung as a result of compression by the fluid and aortic aneurysm (Fig. I). Antibiotics were implemented due to clinical, laboratory and auscultatory symptoms of pneumonia. During hospitalization, melaena and progressive anemia have been occurred (with the marginal level of $\mathrm{Hb} 6 \mathrm{~g} / \mathrm{dl}$ ). Due to the patient's clinical status, the blood products were transfused. CT angiography of aorta showed the presence of gas in aneurysm sac, defect in the aortic wall and thickened esophagus with the level of fluid (Figs 2-4). The whole radiological feature had shown the presence of AEF. Oral water contrast-enhanced X-ray of the esophagus revealed extraesophageal leakage of contrast, most likely into the aneurysmal sac (Fig. 5). EGD under general anesthesia was performed and demonstrated the presence of a wide fistula between aneurysmal sac and the mid-thoracic esophagus. The SEMS prosthesis was implanted under endoscopy control. In addition, control esophagus $x$-ray examination revealed the tightness of the prosthesis (Fig. 6). Open surgical repair of the aneurysm after the patient's general condition improvement was planned. Despite the temporary clinical stabilization and no significant improvement, the patient died 13 days after admission to the hospital.

\section{Case report 2}

66-year-old woman, who had undergone endovascular treatment of the thoracoabdominal aneurysm using stent graft with branches to the visceral arteries

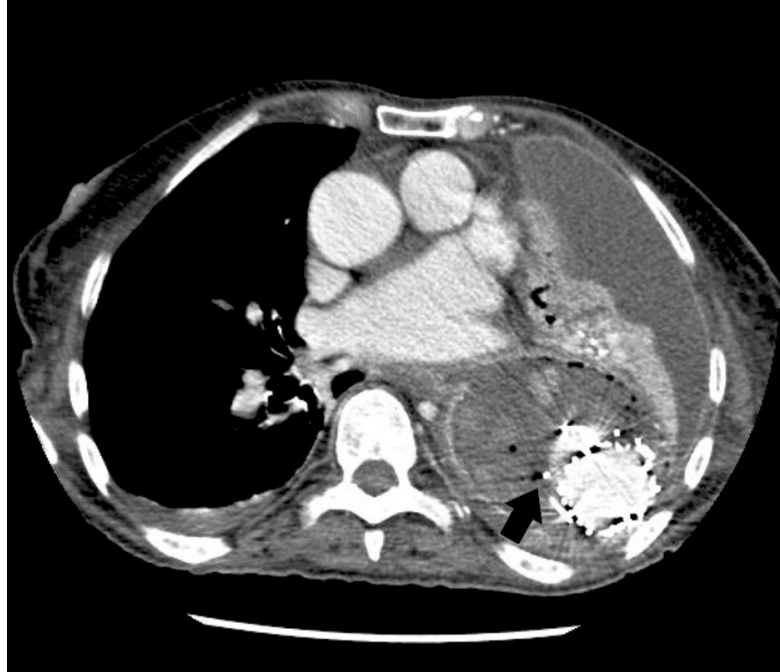

Figure 2. Case report I. Computer tomography showing the presence of gas in the aneurysmal sac (black arrow)

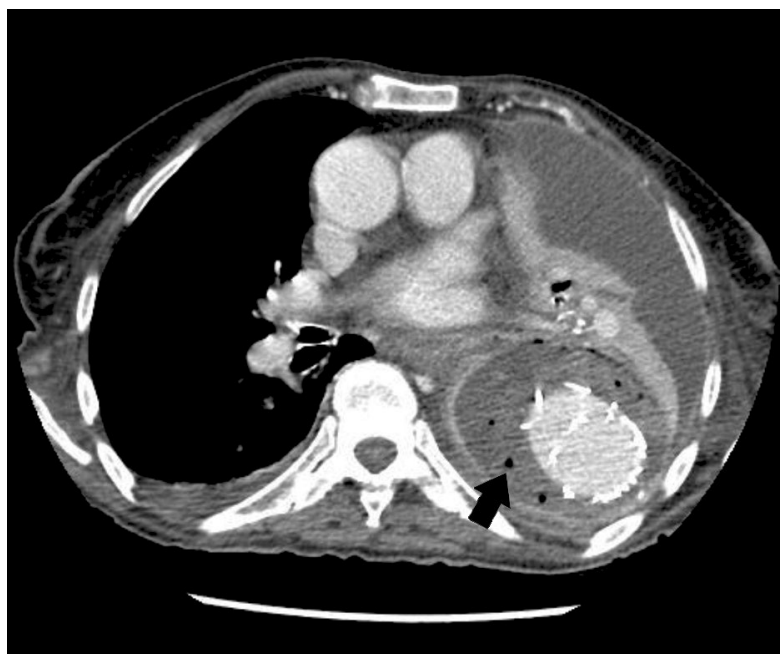

Figure 3. Case report I. Computer tomography showing the presence of gas in the aneurysmal sac (black arrow)

6 months ago, was admitted to the hospital in bad general condition. Based on the angio-CT examination, AEF (defect in the aortic wall and aneurysmal sac), mediastinitis and infection of the aneurysm sac were suspected (Figs 7, 8). The patient had symptoms of dyspnoea, chest pain and fever. On admission, blood tests and blood culture had been taken. Blood biomarkers of bacterial infection were increased (procalcitonin 96 $\mathrm{ng} / \mathrm{ml}$, CRP $328 \mathrm{mg} / \mathrm{dl}$, WBC $12,4 \times 10^{6} \mu \mathrm{l}$ ) and blood culture was positive for Enterococcus species. Antibiotherapy and oxygen therapy had been introduced. Anemia was found with no decline dynamics, which would suggest active bleeding. Taking into considera- 


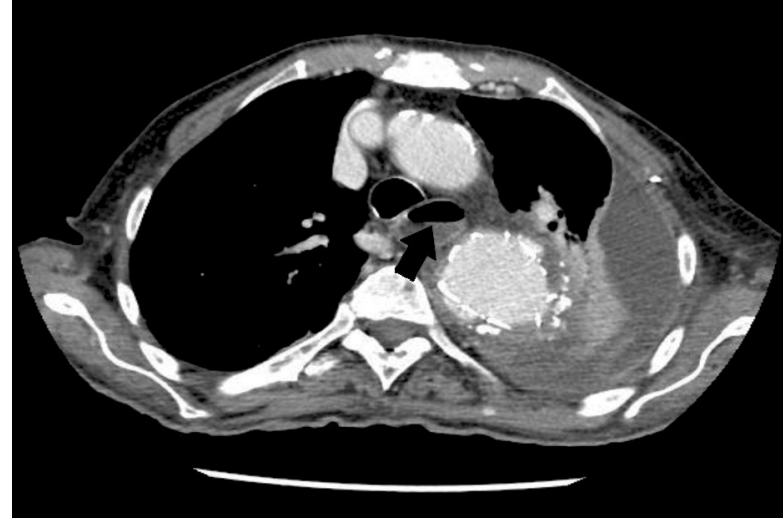

Figure 4. Case report I. Computer tomography showing thickened esophagus with the level of fluid (black arrow)

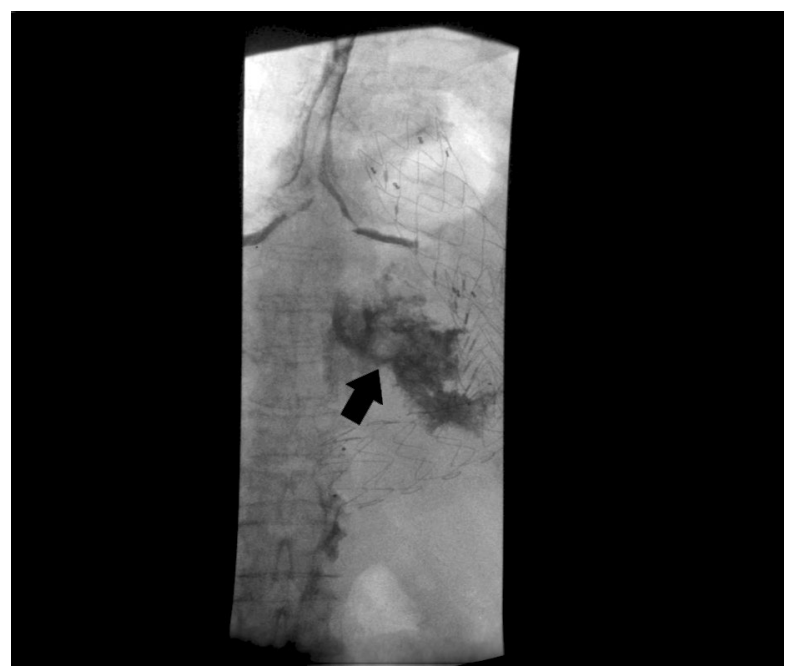

Figure 5. Case report I. Oral water contrast-enhanced X-ray examination of the esophagus showing extraesophageal leakage of contrast (black arrow)

tion bad general condition and no chance of survival, the patient was disqualified from open surgical repair of the thoracoabdominal aneurysm. The patient died 4 days after admission.

\section{Discussion}

Aortoesophageal fistula (AEF) is a rare complication after TEVAR [I, 2]. In the literature, authors underline the great role of quick diagnostics due to difficult to control anemia [2,3]. Computer tomography is an examination of choice in patients who had undergone TEVAR in case of suspected complications. In patients with AEF, CT angiogram shows the presence of gas in aneurysm sac $[2,4,5]$, thickening of esophagus [6], and new nonhomogenous masses between aorta and

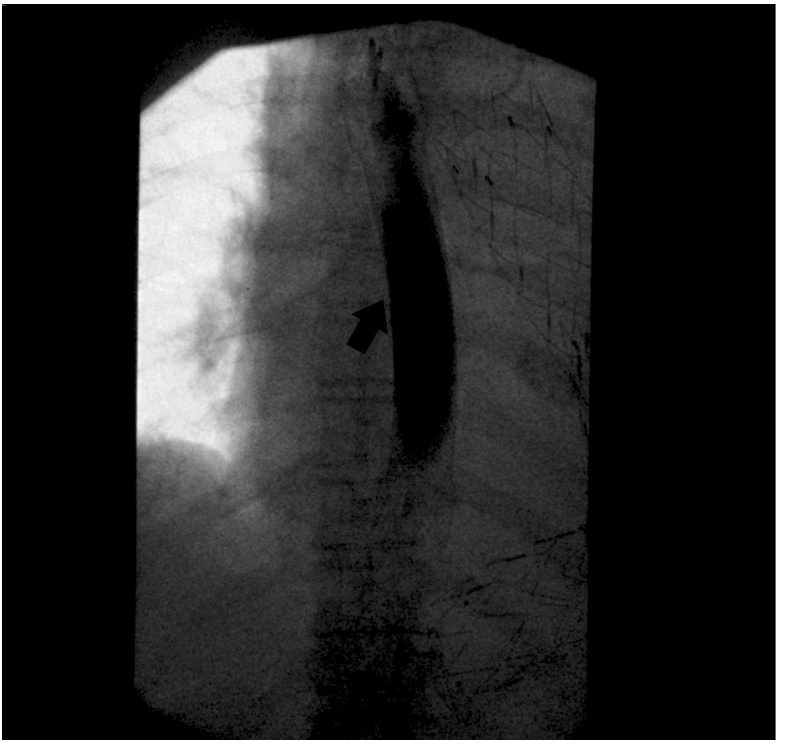

Figure 6. Case report I. Esophagus x-ray examination showing the tightness of the SEMS prosthesis (black arrow)

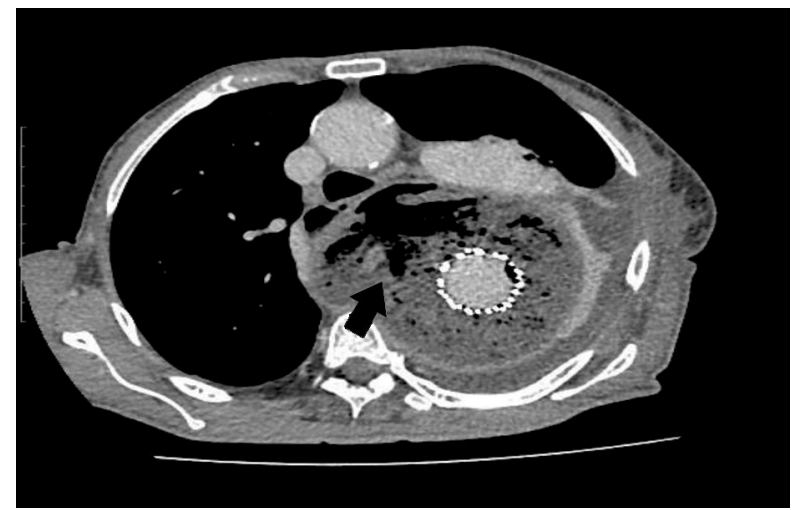

Figure 7. Case report 2. Computer tomography showing the presence of gas in the aneurysmal sac (black arrow)

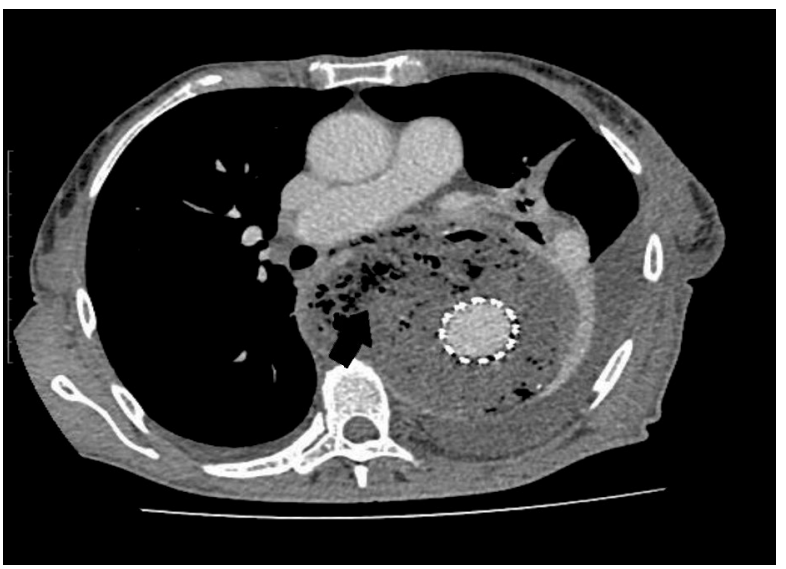

Figure 8. Case report 2. Computer tomography showing the presence of gas in the aneurysmal sac (black arrow) 
esophagus [2, 7]. In our cases, the presence of gas in aneurysm sac (Patient I and 2), as well as thickened esophagus and defect in the aortic wall (Patient I) were detected. Esophagus $x$-ray examination with contrast can confirm or exclude the presence of AEF (as per case I which confirmed preliminary diagnosis based on CT). EGD not always identifies the place and cause of bleeding $[5,6]$. However, in combination with CT scans, it significantly increases the probability of confirmation or exclusion of AEF [2-8]. Therefore, it seems that EGD should be the examination of choice when AEF is suspected, despite the risk of additional transmission of bacteria from the esophagus to the aneurysmal sac.

The time to develop AEF after TEVAR varies. Analyzing literature, we can conclude that AEF develops within I to 16 months after the endovascular procedure [2-8]. Nevertheless, patient I developed AEF much later -5 years after TEVAR.

There are many hypotheses regarding the cause of AEF. Mechanisms of AEF development after TEVAR include: I) pressure necrosis of the esophageal wall due to the ongoing forces of the self-expanding stentgraft, 2) direct erosion of the rigid stent-graft through the aorta into the esophagus, 3 ) ischemic esophageal necrosis due to stent-graft occlusion of aortic side branches that directly feed the esophagus, 4) infection of stent-graft prosthesis and aneurysmal sac that eventually extends to the esophagus eroding its wall $[7,9]$. Due to uncertain pathophysiologic mechanisms, we cannot isolate patients at high-risk, neither the time at which complications may occur.

Patients with AEF after TEVAR usually die before the beginning of treatment due to the presence of bleeding and general bad condition $[3,6,8]$. In these cases, the only option is open surgery with removal of the stent graft and replacement of the aorta with a vascular prosthesis. The majority of patients, such as those described in our article, do not qualify for open surgery $[2,5,5]$. Then, therapeutic opportunities are limited. Therefore, esophageal stent implantation may be palliative procedure $[2,5,7]$. However, this method doesn't provide long-term effectiveness. There was the only one case of a patient with AEF free of the gastrointestinal bleeding reported [7].

Eggebrecht et al. described 6 patients who developed AEF after TEVAR. 4 of 6 patients were admitted to the hospital due to vomiting blood, and 2 other due to elevated markers of inflammation (such as patient $I$ and 2). Surgical repair was performed in only I patient and declined in the remaining because of comorbidities and multiorgan failure. In 2 remaining patients who had undergone EGD for massive hematemesis selfexpanding esophageal stents were implanted in order to control bleeding. Although conservative treatment was applied (percutaneous endoscopy-guided gastrostomy PEG, broad-spectrum antibiotics and proton pump inhibitor), all patients died due to massive bleeding and mediastinitis within 20-22I days after admission to the hospital [2].

Leung reported a 74-year-old patient with a history of TEVAR, who was admitted to the hospital due to recurrent hemoptysis. On admission, the patient wasn't presenting any abnormalities in physical examination, nor in laboratory tests. EGD, bronchoscopy and CT angiography were performed. EGD detected no active bleeding, while bronchoscopy indicated external compression of the distal trachea. CT angiogram revealed the presence of pseudoaneurysm of the thoracic aorta distal to the take-off of the subclavian artery, suggesting vasculitis of the thoracic aorta. Endobronchial ultrasound showed aortic diverticulum in the place of tracheal compression. The whole radiological findings confirmed the presence of aortobronchial fistula (ABF). Therefore, it seems that the presence of the pseudoaneurysm, as well as compression of the airways in patients after TEVAR, may also suggest ABF [10].

During 1998-2013, Mosquera et al. [ I I] reviewed 26 patients with thoracic aorta fistulas ( 18 with aortobronchial, 7 with aortoesophageal, and I with co-existing aortic fistulas to the esophagus and bronchial tree). Authors were analyzing $C T$ findings performed in all patients. The most common CT scan findings (in descending order of frequency) were: an intramural hematoma, an aortic pseudoaneurysm bulge, and bronchial compression. Interestingly, none of the patients with $\mathrm{ABF}$ presented periaortic gas. Both active contrast extravasation and the ectopic gas occurred exclusively in those with AEF and were associated with a worse prognosis. Intramural hematoma, an aortic pseudoaneurysm bulge and bronchial compression were common for those with $\mathrm{AEF}$ and $\mathrm{ABF}$. All the authors underline the key role that $C T$ plays in diagnosing patients with $A E F$ and $A B F$.

\section{Conclusions}

Gastrointestinal bleeding, as well as elevated inflammatory markers and atypical clinical feature after TEVAR may suggest the presence of AEF. CT imaging combined with EGD and gastrointestinal $X$-ray examination may confirm or exclude AEF. Nevertheless, the ineffectiveness of treatment indicates that $A E F$ remains a challenge for the medical team.

\section{Conflict of interest}

None. 


\section{References:}

I. Kulesza J. Evaluation of effectiveness and safety of endovascular treatment of patients with thoracic aorta disease by means of a stent-graft. 20I7; WBC Poznań.

2. Eggebrecht H, Mehta R, Dechene A, et al. Aortoesophageal fistula after thoracic aortic stent-graft placement. JACC: Cardiovascular Interventions. 2009; 2(6): 570-576, doi: 10.1016/j. jcin.2009.03.010.

3. Nazarewicz GV, Jain R. Upper gastrointestinal bleeding caused by aortoesophageal fistula. Clin Gastroenterol Hepatol. 2016; 14(12): A22, doi: 10.1016/j.cgh.2016.08.013, indexed in Pubmed: 27546580.

4. Karb DB, Mansoor E, Sullivan J, et al. Atypical presentation of aortoesophageal fistula without hemorrhage. ACG Case Rep J. 2019; 6(2): e00004, doi: 10.14309/crj.0000000000000004, indexed in Pubmed: 31616713.

5. Rawala MS, Badami V, Rizvi SB, et al. Aortoesophageal fistula: a fatal complication of thoracic endovascular aortic stentgraft placement. Am J Case Rep. 2018; 19: 1258-1261, doi: 10.12659/AJCR.91 I44I, indexed in Pubmed: 30348937.

6. Akin M, Yalcinkaya T, Alkan E, et al. A cause of mortal massive upper gastrointestinal bleeding: aortoesophageal fistula. Med Arch. 2016; 70(I): 79-8I, doi: 10.5455/medarh.2016.70.79-8I, indexed in Pubmed: 26980940.
7. Tao M, Shlomovitz E, Darling G, et al. Secondary aorto-esophageal fistula after thoracic aortic aneurysm endovascular repair treated by covered esophageal stenting. World J Clin Cases. 2016; 4(8): 233-237, doi: 10.12998/wjcc.v4.i8.233, indexed in Pubmed: $275746 / 2$.

8. Akaraviputh T, Sriprayoon T, Prachayakul V, et al. Endoscopic diagnosis of secondary aortoesophageal fistula. Endoscopy. 2008; 40 Suppl 2: E90, doi: 10.1055/s-2007-995549, indexed in Pubmed: 18633903.

9. Hance KA, Hsu J, Eskew T, et al. Secondary aortoesophageal fistula after endoluminal exclusion because of thoracic aortic transection. J Vasc Surg. 2003; 37(4): 886-888, doi: 10.1067/ mva.2003.159, indexed in Pubmed: 12663993.

10. Leung AD, Yamanouchi D. Case report of retrograde in situ fenestration of the thoracic stent graft with reentry device in a patient with aortobronchial fistula. Medicine (Baltimore). 2018; 97(24): el I050, doi: 10.1097/MD.00000000000।1050, indexed in Pubmed: 29901605.

II. Mosquera VX, Marini M, Pombo-Felipe F, et al. Predictors of outcome and different management of aortobronchial and aortoesophageal fistulas. J Thorac Cardiovasc Surg. 2014; I48(6): 3020-6.el, doi: 10.1016/j.jtcvs.2014.05.038, indexed in Pubmed: 24974780. 